\title{
MiniJFil@: A New Safe and Effective Stent for Well-Tolerated Repeated Extracorporeal Shockwave Lithotripsy or Ureteroscopy for Medium-to-Large Kidney Stones?
}

\author{
Benoit Vogt, ${ }^{1,}$ Francois-Noel Desfemmes, ${ }^{1}$ Arnaud Desgrippes, ${ }^{1}$ and Yves Ponsot $^{2}$ \\ ${ }^{1}$ Department of Urology, Blois Polyclinic, La Chaussee Saint-Victor, France \\ ${ }^{2}$ Department of Urology, University of Sherbrooke, Sherbrooke, Canada \\ "Corresponding author: Benoit Vogt, Department of Urology, Blois Polyclinic, La Chaussee Saint-Victor, France. Tel: +33-663220844, Fax: +33-254906566, E-mail: \\ message@benoitvogt.fr
}

Received 2016 July 13; Accepted 2016 August 02.

\begin{abstract}
Background: Percutaneous nephrolithotomy (PCNL) is recommended for treating staghorn stones or stones measuring $>20 \mathrm{~mm}$. Extracorporeal shockwave lithotripsy (ESWL) or flexible ureteroscopy (URS) may be used as a complement. However, PCNL can cause trauma to the kidney parenchyma, and patients may find a noninvasive procedure, such as ESWL, to be more attractive.

Objectives: The aim of this study was to evaluate the clinical efficiency of MiniJFil@ stenting associated with ESWL or second-line URS for the treatment of medium-to-large kidney stones. The MiniJFil@ is a stent reduced to a suture of $0.3 \mathrm{~F}$ attached to a renal pigtail. The entire ureter is occupied only by the suture of the stent.

Methods: We retrospectively analyzed the data of 28 patients. Twenty-four patients had kidney stones measuring $>15 \mathrm{~mm}$ (group 1) and four patients had staghorn stones (group 2). All of the patients were fitted with MiniJFil@ 2 - 3 weeks before any treatment. ESWL was always our first-line therapy. Stone-free (SF) status was defined as no evidence of stones.

Results: In group 1, the mean largest and cumulative stone diameters, respectively, were $18.7 \pm 5.7 \mathrm{~mm}$ and $45.0 \pm 12.0 \mathrm{~mm}$. In group 2 , the mean volume was $6,288.4 \pm 2,733.0 \mathrm{~mm}^{3}$. The overall SF was $96.4 \%$ (100\% for group 1 and $75 \%$ for group 2 ). The mean number of sessions of ESWL and URS, respectively, was $1.4 \pm 0.7$ and $0.8 \pm 0.9$ in group 1 and $4.0 \pm 2.0$ and $1.5 \pm 1.3$ in group 2 . The mean times to achieve these rates were $3.2 \pm 1.7$ months and $5.6 \pm 2.3$ months for groups 1 and 2 , respectively. One patient in group 2 was treated with only three sessions of ESWL. Renal colic was observed in only five patients (17.9\%).

Conclusions: MiniJFil $@$ stenting is safe and may be an alternative for the treatment of kidney stones during minimally invasive procedures.
\end{abstract}

Keywords: Staghorn Stone, Stent, Thread, Dilation, Lithotripsy, Ureteroscopy

\section{Background}

Percutaneous nephrolithotomy (PCNL) is recommended as a first step to treat staghorn stones or stones measuring $>20 \mathrm{~mm}$. Extracorporeal shockwave lithotripsy (ESWL) or flexible ureteroscopy (URS) may be used as a complement (1). However, PCNL can cause trauma to the kidney parenchyma and blood transfusion may be required (2). Moreover, patients find noninvasive procedures, such as ESWL, more attractive $(3,4)$.

After ESWL, the incidence of steinstrasse increases with the stone burden and can reach $40 \%$ for stones measuring $>31 \mathrm{~mm}$. Stenting with a double-pigtail stent reduces steinstrasse (5). However, the double-pigtail stent is poorly tolerated and reduces the patient's quality of life (6-8).

It has been suggested that changes in the size and form of stents could decrease discomfort, and we developed
JFil@ stents as a means of decreasing urinary symptoms (7, $8)$. In these innovative stents, the lower part of the doublepigtail stent is replaced by a $0.3 \mathrm{~F}$ suture thread, significantly decreasing urinary symptom and pain scores and constituting a medical advance in the domain of ureteral stent tolerance. Fortuitously, we discovered that the sutures had surprising properties. First, we observed a clear dilation of the ureter containing the sutures. Secondly, after ESWL, the stone fragments gradually slid down the sutures, without renal colic in most cases $(7,8)$.

These surprising properties led us to create the MiniJFil@, which is reduced to a thread attached to a simple loop of a $4.8 \mathrm{~F}$ pigtail stent. With the suture thread, the entire ureter can be dilated, and the luminal vacuity of the ureter is preserved. Thus, we believe that the use of the MiniJFil $®$ may accelerate removal of stone fragments. 


\section{Objectives}

In this study, we evaluated the clinical efficiency and safety of MiniJFil $@$ stenting associated with minimally invasive procedures for the treatment of kidney stones measuring $>15 \mathrm{~mm}$ and staghorn stones.

\section{Methods}

From April 2013 to October 2015 in a single institution, we retrospectively reviewed the records of 28 patients with non-obstructive kidney stones who had undergone exclusive MiniJFil $\circledast$ stenting before and during minimally invasive procedures.

Among the 28 patients, 24 had kidney stones measuring $>15 \mathrm{~mm}$ (group 1 ) and four had staghorn stones (group 2). No staghorn stones were included in group 1 . To evaluate stone status in group 1, we used the largest stone diameter (LSD), cumulative stone diameter (CSD), and volume (VOL). The stone volume was calculated using the sphere formula $\left(4 / 3^{*} \pi^{*} \mathrm{r} 3\right)$. Computed tomographic (CT) 3D reconstruction allowed volume measurements of staghorn stones (Image Analyser AVA, Philips Brilliance, 16 detector arrays). The CSD is a well-established parameter used most frequently in clinical practice to evaluate stone status; however, for stones measuring $>20 \mathrm{~mm}$, the use of volume is recommended (9).

All of the patients were fitted with the MiniJFil ${ }^{\circ} 2$ - 3 weeks before any treatment. The minimally invasive procedures consisted of ESWL and URS, which were performed by the same surgeons (B.V., F.N.D., and A.D.). ESWL was always our first-line therapy. The patients were informed about the different techniques, and preferred to undergo the minimally invasive procedures. All patients understood that the number of sessions and the duration of treatment would be important.

ESWL was performed with a mobile lithotripter (Compact Sigma, Dornier MedTech, Germany). ESWL was administered on an outpatient basis. All patients were treated with intravenous analgesia, and no anesthesia was required. Stones were radiographically located in two planes and were sometimes located with the inline ultrasound head. The shockwave intensity was 4 , and 2,500 shocks were delivered to each patient. The mean time for one ESWL session was 30 minutes. In our center, only one session of ESWL per month can be offered to each patient.

The URS was performed with direct videoscopic guidance under general anesthesia, with a rigid ureteroscope (12 Fr, 27002 L, Karl Storz, Germany) or a flexible ureteroscope (8.5 Fr, 11278 A, Richard Wolf, Germany), or both. No active dilatation of the ureteral orifice was necessary for the patients with MiniJFil@. The treatment was performed beside the MiniJFil@. Stones were fragmented using a pneumatic lithotripter (Swiss LithoClast $\circledast$ Master, EMS, Switzerland) during rigid URS. Flexible URS was performed after insertion of a ureteral sheath (10-12 Fr, ACXL10, Porges-Coloplast, France). The stones were fragmented using a StoneLightTM laser (Boston Scientific, USA), then removed with a stone basket.

At the end of the operation, the threads of the MiniJFil $\circledast$ should cross the junction between the ureter and the bladder, and float in the bladder or the urethra. After URS, the patients were fitted with an external ureteral stent (5 Fr, Open-End Flexi-Tip $®$, G14521, Cook, USA) beside the threads, overnight following the operation. Two hours before external stent removal, $8 \mathrm{mg}$ of betamethasone was administered intravenously.

ESWL was always our first-line therapy. After the procedure, appropriate medical therapy was offered if renal colic occurred. Abundant fluid intake (3 liters/day) and body gymnastics with head down and feet up were recommended for all patients.

The patients were assessed with X-ray immediately after the procedure and 2 - 4 weeks after an ESWL session. The required number of subsequent sessions of ESWL or URS was determined based on residual stones. The determination of stone-free status was achieved by direct visualization of the kidney with URS or by sonography, non-contrast $\mathrm{CT}$, and X-ray. Stone-free (SF) status was defined as no evidence of stones, and patients who did not meet the criteria required further treatment with ESWL or URS. The MiniJFil $[$ was withdrawn under local anesthesia when the treatment was considered complete. The data are presented as mean \pm SD.

\subsection{Technique: Construction, Implantation, and Ablation of the MiniJFil $₫$}

The MiniJFil $@$ stent (WO2014096264 A1) was previously used in another study [7]. In this procedure, a polyurethane double-pigtail stent (double-loop ureteral stents, $4.8 \mathrm{~F}, 26 \mathrm{~cm}$, Coloplast) is sectioned perpendicularly to the main axis, just outside of the renal loop. The sectioned part is then cut parallel to the main axis to form a beveled tail that is $2 \mathrm{~cm}$ long. The tail is then thinned out toward the lower end, where its diameter should not exceed $0.5 \mathrm{~mm}$. The length of the tail and the thinning out of the lower end are important in order to limit the catching of the stent on the junction and the ureter during stent removal. A polypropylene suture (Ethicon monofilament polypropylene suture; gauge size U.S.P.1; 0.1 to $0.15 \mathrm{~mm}$; 5 - 0) perforates the loop and the end of the tail, and a knot is tied at the loop. The distal end of the MiniJFil ${ }^{\circ}$ consists of two $0.3 F$ sutures, each suture approximately $36 \mathrm{~cm}$ long. 
This stent has a total length of $38 \mathrm{~cm}$. Figure 1 shows the different sizes between native $4.8 \mathrm{~F}$ pigtail stents and the $0.3 \mathrm{~F}$ sutures.

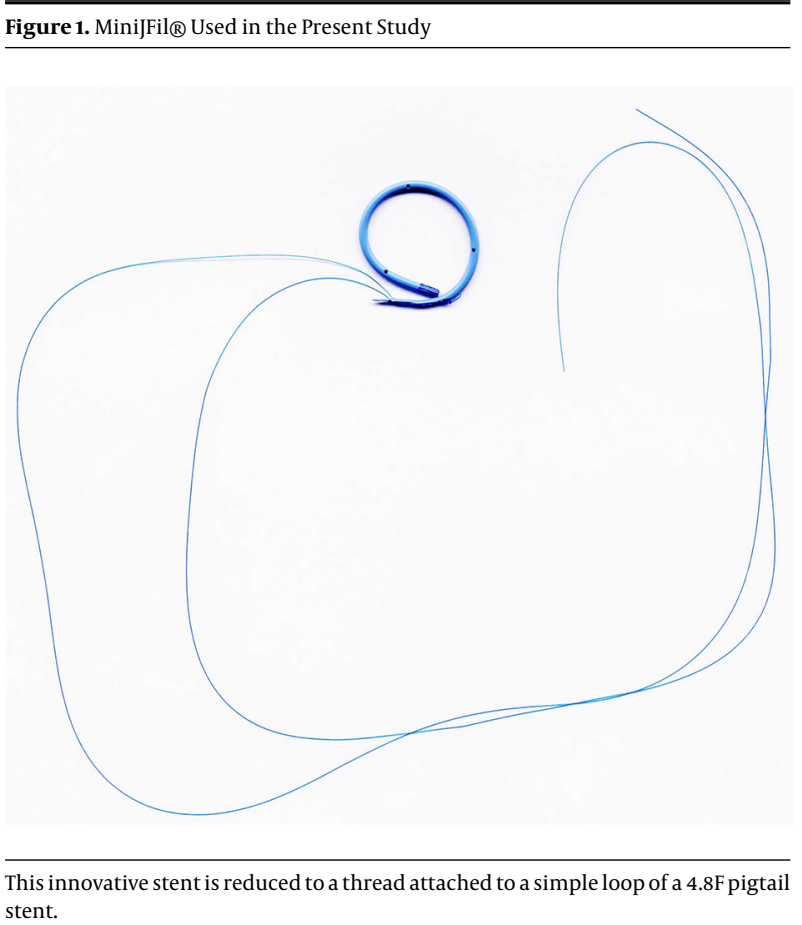

\subsection{Ethics Statement}

The study design was approved by the French ethical committee, Comite de Protection des Personnes (CPP), Ile de France 2 (IRB registration 00001072). The local ethics committee also provided approval. Methods were carried out in accordance with the approved guidelines. Written information was provided to all patients, and all patients gave their informed consent.

\section{Results}

The age, sex, side of stone, stone specifications, localization, number of procedures, and outcomes for the patients are summarized in Table 1 . In group 1, five patients had multiple stones, with an average of 2.2 stones per kidney, and preoperative measurements of stone burden were assessed in 16 non-contrast CTs and 12 X-rays. Mean LSD, CSD, and VOL, respectively, were $18.7 \pm 5.7 \mathrm{~mm}, 45.0 \pm 12.0$ $\mathrm{mm}$, and $1,907.8 \pm 1,876.2 \mathrm{~mm}^{3}$. In group 2 , mean VOL was $6,288.4 \pm 2,733.0 \mathrm{~mm}^{3}$.

The overall SF rate was $96.4 \%$ (100\% for group 1 and $75 \%$ for group 2). One patient from group 2 (Figure $2 \mathrm{~B}$ ) had an asymptomatic stone on sonography (LSD: $7 \mathrm{~mm}$ ). The
Table 1. Patients, Kidney Stone Details, and Outcomes

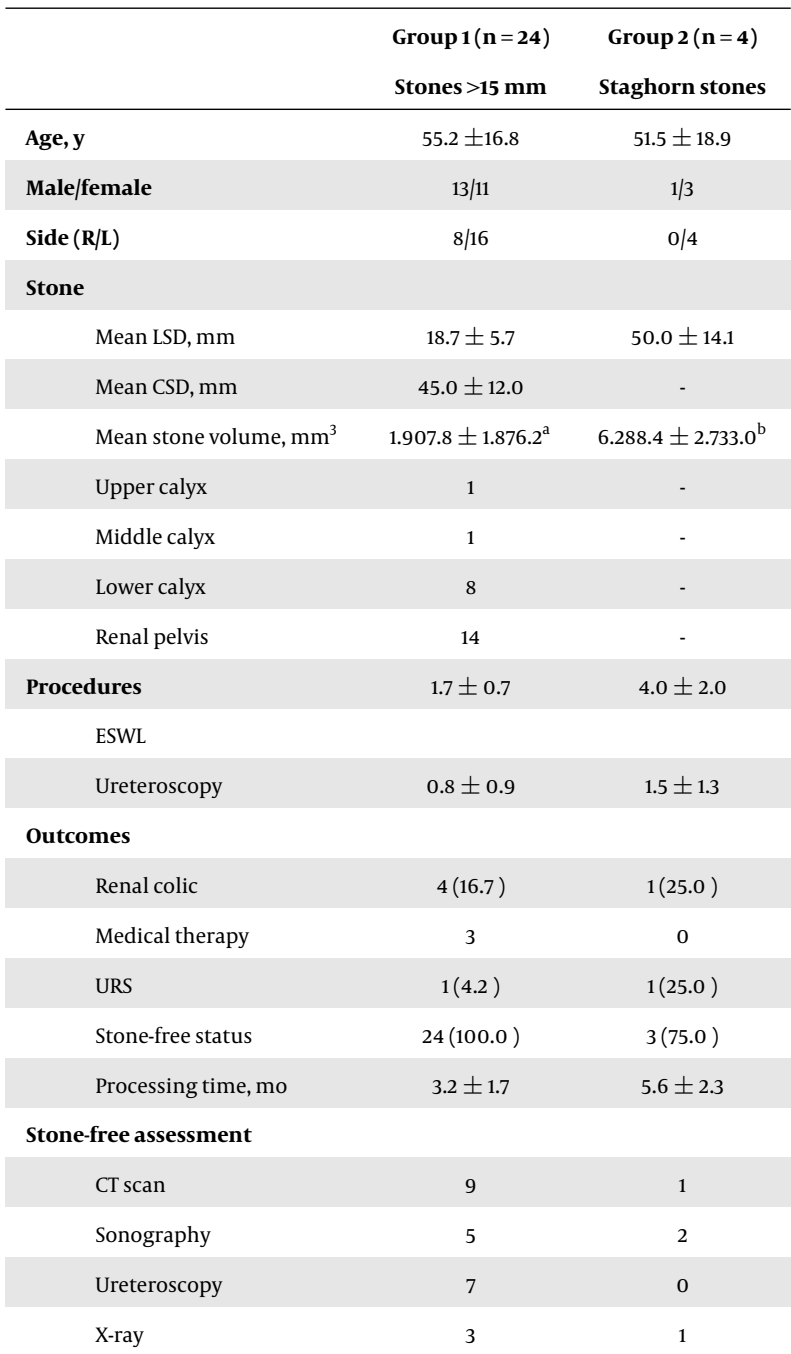

Abbreviations: CSD, cumulative stone diameter; CT, computed tomography; ESWL, extracorporeal shockwave lithotripsy; LSD: largest stone diameter.

${ }^{a}$ Mean stone volume calculated using the sphere formula $\left(4 / 3^{*} \pi^{*} \mathrm{r} 3\right)$.

${ }^{\mathrm{b}}$ Mean stone volume calculated using 3D CT reconstruction.

mean number of ESWL sessions was $1.4 \pm 0.7$ for group 1 and $4.0 \pm 2.0$ for group 2 . The mean number of URS sessions was $0.8 \pm 0.9$ for group 1 and $1.5 \pm 1.3$ for group 2 . The mean time to achieve these rates was $3.2 \pm 1.7$ months for group 1 and $5.6 \pm 2.3$ months for group 2 .

Ten patients from group 1 were treated only by ESWL. In this subgroup, mean LSD, CSD, and VOL, respectively, were $18.8 \pm 6.5 \mathrm{~mm}, 46.6 \pm 10.8 \mathrm{~mm}$, and 1,975.0 $\pm 1,264.5 \mathrm{~mm}^{3}$. The mean number of ESWL sessions was $1.7 \pm 0.9$ and the mean time for SF status was $2.4 \pm 1.0$ months. In three patients, renal colic occurred and was treated only by medical therapy. One patient from group 2 (Figure 2A) was treated 
with only three ESWL sessions (VOL: 4,225 $\mathrm{mm}^{3}$ ).

In five patients (17.9\%), renal colic was observed. Three patients were treated with medical therapy and two with URS. In the case of one patient from group 1, MiniJFil $®$ was replaced with a double-pigtail stent after URS because of a finding of ureteropelvic junction stenosis. Calcifications were encountered around the bladder thread in two patients. No major complications, such as perforation or gross hematuria, were encountered (Figure 3).

\section{Discussion}

The introduction of ESWL in the early 1980s revolutionized the treatment of renal stones (10). However, PCNL is recommended as a first step to treat staghorn stones or stones measuring $>20 \mathrm{~mm}$ (1). Technical developments in endoscopic techniques for the treatment of kidney stones have led to considerable changes in treatment modalities. Moreover, changes in the mechanical design of ESWL could reduce the success rate (11). Recent years have seen a shift from ESWL to endoscopic techniques or PCNL. PCNL may be effective for the treatment of medium-sized kidney stones $(1,12)$, and URS may be effective for the treatment of large and complex kidney stones (13). ESWL remains the standard for stones measuring $<20 \mathrm{~mm}$, and may be used as a complement to other procedures for large stones (1). Thus, ESWL is not recommended for large stones due to an increased incidence of steinstrasse, and double-pigtail stenting does not prevent steinstrasse (5). Moreover, the doublepigtail stent is poorly tolerated and reduces the patient's quality of life (6-8).

In previous studies, we discovered fortuitously that sutures had surprising, unknown properties in the ureter. In all cases, we observed clear dilation of ureters containing sutures, with a ureteral diameter two to three times larger than the contralateral ureter on CT. No inflammation was visible in the ureteral or bladder mucosa. After ESWL, the stone fragments gradually slid down the sutures, without renal colic in most cases $(7,8)$. These unexpected properties naturally led us to expand the list of indications for ESWL and to include staghorn stones.

Complications from ESWL are rarely encountered, with rates of $2.9 \%$ to $7.1 \%$ (14-17). Significant complications are associated with PCNL, with rates of $16 \%$ to $28.5 \%(2,18)$, and these especially include the risk of blood loss requiring transfusion. In our practice, minimally invasive procedures, such as ESWL, are always our first-line therapy. Moreover, patient preferences must be considered. Kuo et al. and, recently, Raja et al. have pointed out that patients preferentially chose ESWL rather than invasive procedures, such as PCNL $(3,4)$.
The SF rate is defined as no evidence of stones (15), and the clinical success (CS) rate is defined as the presence of stone fragments measuring $<4 \mathrm{~mm}(12-15,19)$. In most of the referenced studies, patients were assessed after three months.

The probability of renal stone clearance after ESWL decreased with increasing stone size in all locations $(14,16)$. Tan et al. reported a SF rate of 53\% and a CS rate of $81 \%$ at three-month follow-up for the ESWL treatment of 1,666 renal stones. For renal stones measuring $>20 \mathrm{~mm}$, the SF and CS rates depended on their location in the kidney, and were $31 \%-52 \%$ and $44 \%$ - 63\%, respectively. For lower-pole calyceal stones, the SF rate was only 31\% (14). However, the best results were obtained by the urologist who treated the greatest number of patients (17).

Interestingly, Fall et al. reported a similar CS rate of $52.7 \%$ for renal stones measuring $>20 \mathrm{~mm}$ and treated by URS (19). Better results were reported by Cohen et al. for renal stones measuring 19 - $37 \mathrm{~mm}$, with CS of $81 \%$ - 94\% (13).

The incidence of steinstrasse increases with the stone burden, and staghorn stones are unsuitable for ESWL monotherapy $(5,14,20)$. In the treatment of staghorn stones, Yan et al. reported a SF rate of 53.2\% with one session of PCNL, and of $82.9 \%$ after multiple procedures (PCNL, ESWL, URS) (18). Good results were reported by Netto with multiple procedures (ESWL and URS), for a CS rate of $82.4 \%$ (2).

In the present study, we achieved SF rates of $100 \%$ and $75 \%$ in groups 1 and 2, respectively. While our results are better than those of other reported series, our study was limited by a low number of patients. Moreover, the outcomes were assessed after more than three months. In the case of staghorn stones, ESWL was always our first-line therapy, and we cannot now compare our results to those of other series. However, ESWL and URS during MiniJFil ${ }^{\circledR}$ stenting seem to be a possible treatment for large stones. One patient from group 2 with a staghorn stone was successfully treated with ESWL alone.

Several factors could explain the SF rates in the present study:

- Our mobile ESWL device is available only monthly. Several studies showed that elimination of stones may increase with postoperative time (20-22). In our study, the better tolerance of MiniJFil@ led us to wait for the elimination of all fragments (7).

- In comparison with the double-pigtail stent, the luminal burden of the ureter was reduced to the threads of the MiniJFil $®$. Moreover, the MiniJFil $®$ allowed dilation of the ureter without any inflammation (7). Sfoungaristos et al. showed that ureteral stents, even if they were removed just before ESWL, decreased the SF rate. Edema formation with decreasing functional ureteral lumen diameter and low 

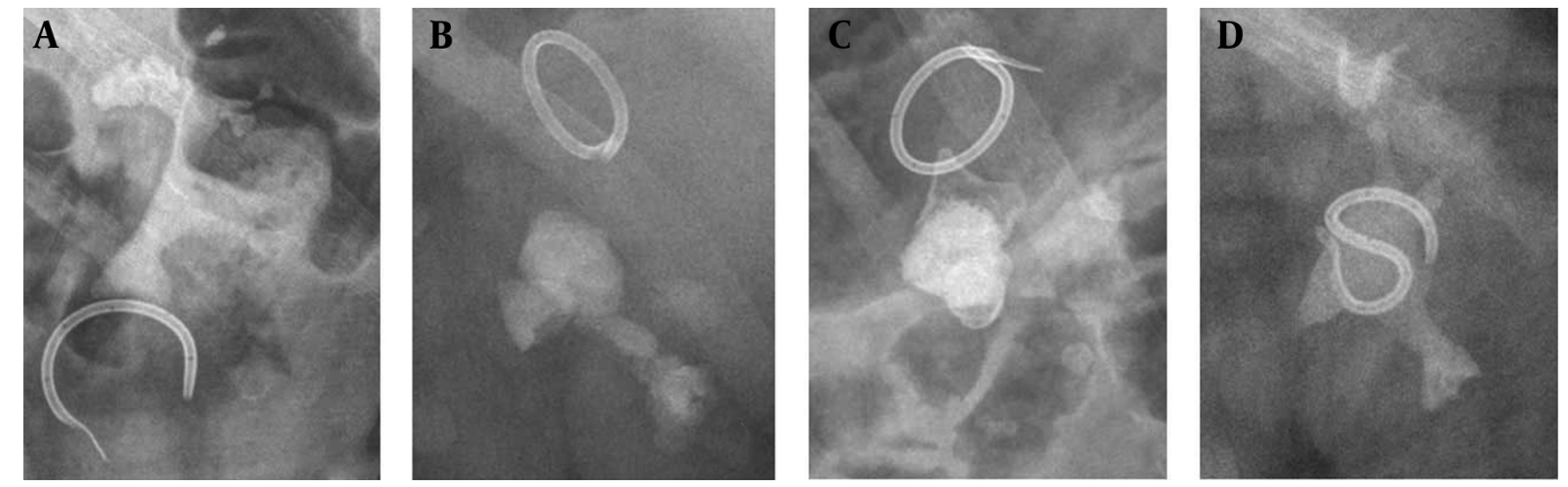

Figure 2. Appearance of Staghorn Stones and MiniJFil@ on X-Ray

Figure 3. Appearance of a 24-mm Renal Stone on X-ray During Treatment With Extracorporeal Shockwave Lithotripsy (ESWL) and MiniJFil@
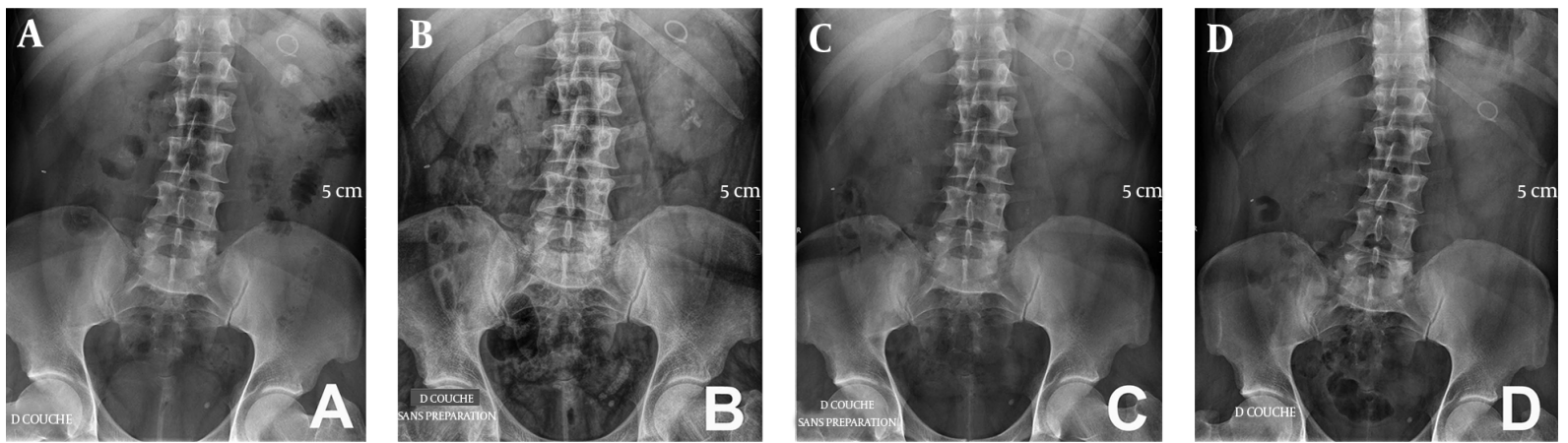

A, MiniJFil $®$ and stone before ESWL; B, stone fragmentation and steinstrasse on day 4 after ESWL; C, spontaneous evacuation of steinstrasse on day 10 after ESWL; D, complete evacuation of the rest of the stone on day 28 after ESWL.

ureteral peristalsis may minimize the likelihood of stone passage $(23,24)$. The absence of edema around the suture may facilitate stone elimination. Thus, luminal freedom with ureteral dilatation may allow elimination of the stone fragments.

- In a previous study, in addition to ureteral dilatation, pelvic and calyceal dilation was observed in $50 \%$ of cases after MiniJFil@ stenting (7). Brewer et al. showed that luminal flow in the stent increased with the internal diameter of the lumen (25). A better ureteral flow, thanks to abundant fluid intake, may facilitate mobilization and elimination of stone fragments.

- Finally, we always recommended postural gymnastics. It has been shown that gymnastics associated with hydrotherapy was effective for the elimination of stone fragments after ESWL (26).

The SF rate, which was the main objective of these studies, did not take into account the patient's quality of life. Although the complication rates of ESWL were lim- ited, they concerned the immediate complications of procedures (14-17). Few studies have so far attempted to describe the patient's quality of life over long-term follow-up after ESWL.

Sahin et al. evaluated the possible effects of residual fragments on quality of life in 71 patients after ESWL. In the first month following ESWL, $40.5 \%$ of patients with stones measuring $<4 \mathrm{~mm}$ and $69.0 \%$ of patients with stones of $>4 \mathrm{~mm}$ had renal colic. Additional procedures were required for $40.8 \%$ of the patients (21). Streem et al. determined the natural history and the clinical implications of 160 patients with stones of $<4 \mathrm{~mm}$ after ESWL for a mean period of 23 months. Renal colic was observed in $43.1 \%$ of the patients, and additional procedures were required in 27.5\% (27). Even after URS, additional procedures were required for $8.2 \%-46.2 \%$ of patients for residual fragments over a period of five years (28). Hubner et al. suggested that $39 \%$ of asymptomatic renal calyceal stones were increased in size, and $83 \%$ of patients required surgical procedures 
within five years of diagnosis (29). Meanwhile, Kelley et al. observed that prophylactic ESWL for asymptomatic renal calyceal stones measuring $<15 \mathrm{~mm}$ reduced the risk of invasive procedures in cases of renal colic over a mean followup of two years (30). Several studies have shown that elimination of stones may increase with postoperative time (2022). In our study, the better tolerance of MiniJFil $®$ led us to wait for the elimination of all fragments, without excessive discomfort for the patient (7).

In the present study, only five out of 28 patients (17.9\%) had renal colic during the observation period, and two patients (7.1\%) required an additional procedure (URS). In group 2, it was remarkable that only one case of renal colic occurred during the 5.6 months of treatment. MiniJFil $®$ stenting seems to limit renal colic, as we have previously observed, even when steinstrasse occurs (7).

MiniJFil $®$ stenting with ESWL is not intended to replace other techniques, but provides a minimally invasive alternative treatment. ESWL is not recommended for staghorn stones, but the potential of MiniJFil $®$ led us to reassess the list of indications.

There were several limitations to this study due to its retrospective character and low number of patients. Another limitation was that the study included only a single medical center. Further studies from multiple centers in a randomized, controlled trial should confirm the improvements in treatment reported here. The evaluation of SF status in the present study can also be criticized because the visualization of small stones may be difficult. In this study, four patients were assessed with plain abdominal X-rays, and SF status may have been overestimated.

Based on our experience with 580 JFil $®$ and MiniJFil $®$ stentings, we can declare other limitations to the use of MiniJFilß. First, the ureter must always be healthy in its entirety. In the presence of inflammatory or fibrous stenosis or post-radiation stenosis, MiniJFil $®$ will be ineffective for dilation and for the descent of the stone fragments. In group 1 in this study, one patient had moderate ureteropelvic junction stenosis; future MiniJFil@ stenting is clearly not recommended for this patient. Second, the thread is poorly effective in the presence of postoperative edema and blood clots. For this reason, we used an external ureteral stent beside the MiniJFil@ thread during the night following the operation whenever URS proved difficult. Third, in cases of less-abundant fluid intake, hyperuricemia, or hypercalcemia, calcifications may occur around the thread, especially in the bladder portion of the sutures. Abundant drinking of liquids is recommended for all patients.

The MiniJFil $®$ will be commercialized in 2016 by ASPIDE® MEDICAL, and further studies should reveal further properties of the ureteral thread. For example, prior im- plantation of a MiniJFil $®$ could be used to prepare the ureter for the insertion of a sheath for flexible URS, without excessive discomfort for the patient $(7,8)$. Ureteral dilation could facilitate the introduction of a large endoscope (11F) for ureteroscopic treatment of large stones (31).

\subsection{Conclusions}

MiniJFil $\circledast$ stenting is safe and seems to be an alternative for the treatment of medium-to-large kidney stones during minimally invasive procedures. ESWL is not recommended for large stones, but the potential of MiniJFil $®$ led us to reassess the list of indications. In this study, we present a new method for the treatment of renal stones. MiniJFil@ stenting could change current endourology techniques by providing greater patient comfort and improvements in the daily practice of urologists.

\section{Acknowledgments}

We thank Dr. Loïc Besnier, radiologist, Polyclinique de Blois, for his calculations of stone burden on CT images, and Professor Janine Dove-Rumé, English Department at the University of Tours, for re-reading our text.

\section{Footnote}

Authors' Contribution: Benoit Vogt and Francois-Noel Desfemmes developed the original idea. Benoit Vogt developed the protocol, analyzed data, and wrote the manuscript. All authors approved the manuscript's content before submission.

\section{References}

1. Turk C, Petrik A, Sarica K, Seitz C, Skolarikos A, Straub M, et al. EAU Guidelines on Interventional Treatment for Urolithiasis. Eur Urol. 2016;69(3):475-82. doi: 10.1016/j.eururo.2015.07.041. [PubMed: 26344917].

2. Netto NJ, Ikonomidis J, Ikari O, Claro JA. Comparative study of percutaneous access for staghorn calculi. Urology. 2005;65(4):659-62. doi: 10.1016/j.urology.2004.10.081. [PubMed: 15833502] discussion 662-3.

3. Kuo RL, Aslan P, Abrahamse PH, Matchar DB, Preminger GM. Incorporation of patient preferences in the treatment of upper urinary tract calculi: a decision analytical view. JUrol. 1999;162(6):1913-8. [PubMed: 10569536] discussion 1918-9.

4. Raja A, Hekmati Z, Joshi HB. How Do Urinary Calculi Influence HealthRelated Quality of Life and Patient Treatment Preference: A Systematic Review. J Endourol. 2016;30(7):727-43. doi: 10.1089/end.2016.0110. [PubMed: 27080725].

5. Al-Awadi KA, Abdul Halim H, Kehinde EO, Al-Tawheed A. Steinstrasse: a comparison of incidence with and without J stenting and the effect of J stenting on subsequent management. BJU Int. 1999;84(6):618-21. [PubMed: 10510104]. 
6. Joshi HB, Stainthorpe A, MacDonagh RP, Keeley FJ, Timoney AG, Barry MJ. Indwelling ureteral stents: evaluation of symptoms, quality of life and utility. J Urol. 2003;169(3):1065-9. doi: 10.1097/01.ju.0000048980.33855.90. [PubMed: 12576847] discussion 1069.

7. Vogt B, Desgrippes A, Desfemmes FN. [Pigtail suture stent: decisive progress towards double-pigtail stent tolerance and unexpected properties of the suture in the ureter]. Prog Urol. 2014;24(7):441-50. doi: 10.1016/j.purol.2013.12.007. [PubMed: 24861684].

8. Vogt B, Desgrippes A, Desfemmes FN. Changing the double-pigtail stent by a new suture stent to improve patient's quality of life: a prospective study. World J Urol. 2015;33(8):1061-8. doi:10.1007/s00345014-1394-2. [PubMed: 25213219].

9. Merigot de Treigny O, Bou Nasr E, Almont T, Tack I, Rischmann P, Soulie $M$, et al. The Cumulated Stone Diameter: A Limited Tool for Stone Burden Estimation. Urology. 2015;86(3):477-81. doi: 10.1016/j.urology.2015.06.018. [PubMed: 26135811].

10. Chaussy C, Brendel W, Schmiedt E. Extracorporeally induced destruction of kidney stones by shock waves. Lancet. 1980;2(8207):1265-8. [PubMed: 6108446].

11. Jain A, Shah TK. Effect of air bubbles in the coupling medium on efficacy of extracorporeal shock wave lithotripsy. Eur Urol. 2007;51(6):1680-6. doi: 10.1016/j.eururo.2006.10.049. [PubMed: 17112655] discussion 1686-7.

12. Wilhelm K, Hein S, Adams F, Schlager D, Miernik A, Schoenthaler M. Ultra-mini PCNL versus flexible ureteroscopy: a matched analysis of analgesic consumption and treatment-related patient satisfaction in patients with renal stones 10-35 mm. World J Urol. 2015;33(12):2131-6. doi: 10.1007/s00345-015-1585-5. [PubMed: 25971205].

13. Cohen J, Cohen S, Grasso M. Ureteropyeloscopic treatment of large, complex intrarenal and proximal ureteral calculi. BJU Int. 2013;111(3 Pt B):E127-31. doi: 10.1111/j.1464-410X.2012.11352.x. [PubMed: 22757752].

14. Tan YM, Yip SK, Chong TW, Wong MY, Cheng C, Foo KT. Clinical experience and results of ESWL treatment for 3,093 urinary calculi with the Storz Modulith SL 20 lithotripter at the Singapore general hospital. Scand J Urol Nephrol. 2002;36(5):363-7. doi: 10.1080/003655902320783872. [PubMed: 12487741].

15. De Sio M, Autorino R, Quarto G, Mordente S, Giugliano F, Di Giacomo F, et al. A new transportable shock-wave lithotripsy machine for managing urinary stones: a single-centre experience with a dual-focus lithotripter. BJU Int. 2007;100(5):1137-41. doi: 10.1111/j.1464410X.2007.07039.x. [PubMed: 17550410].

16. Neisius A, Wollner J, Thomas C, Roos FC, Brenner W, Hampel C, et al. Treatment efficacy and outcomes using a third generation shockwave lithotripter. BJU Int. 2013;112(7):972-81. doi: 10.1111/bju.12159. [PubMed: 24118958].

17. Logarakis NF, Jewett MA, Luymes J, Honey RJ. Variation in clinical outcome following shock wave lithotripsy. J Urol. 2000;163(3):721-5. [PubMed: 10687964].
18. Yan S, Xiang F, Yongsheng S. Percutaneous nephrolithotomy guided solely by ultrasonography: a 5-year study of $>700$ cases. BJU Int. 2013;112(7):965-71. doi: 10.1111/bju.12248. [PubMed: 23889729].

19. Fall B, Mouracade P, Bergerat S, Saussine C. [Flexible ureteroscopy and laser lithotripsy for kidney and ureter stone: indications, morbidity and outcome]. Prog Urol. 2014;24(12):771-6. doi: 10.1016/j.purol.2014.06.007. [PubMed: 25158327].

20. Sayed MA, el-Taher AM, Aboul-Ella HA, Shaker SE. Steinstrasse after extracorporeal shockwave lithotripsy: aetiology, prevention and management. BJU Int. 2001;88(7):675-8. [PubMed:11890235].

21. Sahin C, Tuncer M, Yazici O, Horuz R, Cetinel AC, Eryildirim B, et al. Do the residual fragments after shock wave lithotripsy affect the quality of life?. Urology. 2014;84(3):549-54. doi: 10.1016/j.urology.2014.05.012. [PubMed: 25168532].

22. Resit-Goren M, Dirim A, Ilteris-Tekin M, Ozkardes H. Time to stone clearance for ureteral stones treated with extracorporeal shock wave lithotripsy. Urology. 2011;78(1):26-30. doi: 10.1016/j.urology.2010.10.060. [PubMed: 21333330].

23. Sfoungaristos S, Gofrit ON, Pode D, Landau EH, Yutkin V, Latke A, et al. History of Ureteral Stenting Negatively Affects the Outcomes of Extracorporeal Shockwave Lithotripsy. Results of a Matched-pair Analysis. Prague Med Rep. 2015;116(3):225-32. doi:10.14712/23362936.2015.62. [PubMed: 26445394].

24. Natalin RA, Hruby GW, Okhunov Z, Singh H, Phillips CK, Humphrey PA, et al. Pilot study evaluating ureteric physiological changes with a novel 'ribbon stent' design using electromyographic and giant magnetoresistive sensors. BJU Int. 2009;103(8):1128-31. doi: 10.1111/j.1464410X.2008.08184.x. [PubMed: 19040534].

25. Brewer AV, Elbahnasy AM, Bercowsky E, Maxwell KL, Shalhav AL, Kahn SA, et al. Mechanism of ureteral stent flow: a comparative in vivo study. J Endourol. 1999;13(4):269-71. doi: 10.1089/end.1999.13.269. [PubMed: 10405904].

26. Thomas J. [Hydroposturotherapy and residual lithiasis after lithotripsy]. Prog Urol. 2000;10(6):1152-5. [PubMed: 11217551].

27. Streem SB, Yost A, Mascha E. Clinical implications of clinically insignificant store fragments after extracorporeal shock wave lithotripsy. $J$ Urol. 1996;155(4):1186-90. [PubMed: 8632527].

28. Portis AJ, Laliberte MA, Heinisch A. Repeat Surgery After Ureteroscopic Laser Lithotripsy With Attempted Complete Extraction of Fragments: Long-term Follow-up. Urology. 2015;85(6):1272-8. doi: 10.1016/j.urology.2015.03.019. [PubMed: 26099873].

29. Hubner W, Porpaczy P. Treatment of caliceal calculi. $\mathrm{Br} J$ Urol. 1990;66(1):9-11. [PubMed: 2393803].

30. Keeley FX, Tilling K, Elves A, Menezes P, Wills M, Rao N, et al. Preliminary results of a randomized controlled trial of prophylactic shock wave lithotripsy for small asymptomatic renal calyceal stones. BJU Int. 2001;87(1):1-8.

31. Vogt B, Desgrippes A, Desfemmes F. [Not Available]. Prog Urol. 2014;24(13):896. doi:10.1016/j.purol.2014.09.018. [PubMed: 26461776]. 